\title{
Large Hemangioma in a Persistent Left Superior Vena Cava
}

\author{
Wen $\mathrm{Hu}^{1}$ Xiang Wang ${ }^{1}$ Sichuang $\operatorname{Tan}^{1} \quad$ Songqing Fan ${ }^{1} \quad$ Jun Liu ${ }^{1} \quad$ Fenglei Yu${ }^{1} \quad$ jingqun Tang ${ }^{1}$ \\ ${ }^{1}$ Department of Thoracic and Cardiovascular Surgery, The Second \\ Xiangya Hospital of Central South University, Changsha, Hunan, \\ China \\ Thorac Cardiovasc Surg 2012;60(S2):e3-e5.

\begin{abstract}
Address for correspondence Jingqun Tang, MD, PhD, Department of Thoracic and Cardiovascular Surgery, The Second Xiangya Hospital of Central South University, 139 Renmin Road, Changsha, Hunan 410011,
\end{abstract} \\ China (e-mail: jingquntang@hotmail.com).
}
Abstract
Keywords
- venous disease
- tumor
- cardiopulmonary bypass

Cardiac hemangiomas represent 1 to $2 \%$ of all detected benign heart tumors. Tumors in the coronary sinus have been reported; however, to our knowledge, there have been no reports of masses in a persistent left superior vena cava. We report here the first case of a 58-year-old man with a rare huge unicamerate cardiac hemangiomas in a persistent left superior vena cava. A communication vein between the coronary sinus and hemangiomas could be identified, and thrombus formation was found in the hemangiomas as well.

\section{Introduction}

A 58-year-old man presented to our hospital in December 2010, with main complaints of intermittent cough, chest discomfort of 1 month's duration. Physical examinations and laboratory results showed no abnormality. Transthoracic echocardiography demonstrated an irregular mass in pericardial chamber, with the echo dense mass measuring $108 \times 75 \times 60 \mathrm{~mm}$. Furthermore, inside the mass, a ball-like brightly echogenic core, $60 \times 60 \times 50 \mathrm{~mm}$, was discovered. It indicated that tight relation existed between the right atrium and inferior vena cava ( - Fig. 1a). However, color Doppler flow imaging found no blood flow inside the mass. These findings were also confirmed by computed tomography (CT) (-Fig. 1b).

During the surgery, inside the pericardium, a huge unicamerate cardiac hemangioma in a persistent left superior vena cava was revealed $(\boldsymbol{-}$ Fig. 1c). There was a communication vein of $3 \mathrm{~mm}$ diameter between the coronary sinus and

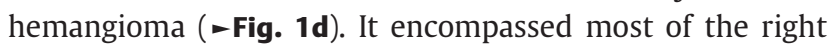
atrium wall and appeared to involve part of the interatrial groove. As the hemangioma had tight relations with the right atrium, cardiopulmonary bypass (CPB) which connected inferior vena cava to descending aorta was established before the persistent left superior vena cava was ligated. The entire hemangioma was carefully excised from the right atrial appendage. The tumor measured $110 \times 65 \times 45 \mathrm{~mm}$ with some white spots on the wall (-Fig. 2a). Pathology revealed a large venous hemangioma (-Fig. $\mathbf{2 b}$ ) with a huge not-organized onion-like-shaped thrombus core ( $\mathbf{- F i g}$. $\mathbf{2 c}$ ). The wall of the mass was composed of flattened endothelial cells, smooth muscle, elastic fibers, and calcified plaques (-Fig. 2d) which demonstrated that was an intravenous hemangioma.

The patient remained stable and was discharged from hospital 10 days after operation. Transthoracic echocardiography showed no remnant of hemangioma.

Cardiac hemangioma is a rare primary benign cardiac tumor, with 1 to $2 \%$ of all detected benign heart tumors which incidence rate is $\sim 0.001$ to $0.03 \%$ at autopsy. ${ }^{1}$ They could be found at any age and occurred in any part of the heart, including the endocardium, myocardium, or pericardium.

The clinical symptoms of cardiac hemangioma are various, from asymptomatic to sudden cardiac death, which are mostly dependent on the size and the anatomic location of the neoplasm. In this case, the initial diagnose was a cyst of pericardium, cardiac hemangioma was identified until pericardium was opened.

The development of imaging techniques help to identify cardiac hemangioma. For its advantages of simple, convenience, safe, and noninvasive, transthoracic echocardiography has been employed as an important and initial tool in received

May 3, 2012

accepted after revision

June 13, 2012

published online

November 23, 2012 (c) 2012 Georg Thieme Verlag KG Stuttgart · New York
DOI http://dx.doi.org/ 10.1055/s-0032-1330995. ISSN 2194-7635. 
e4 Large Hemangioma in a Persistent Left Superior Vena Cava Hu et al.

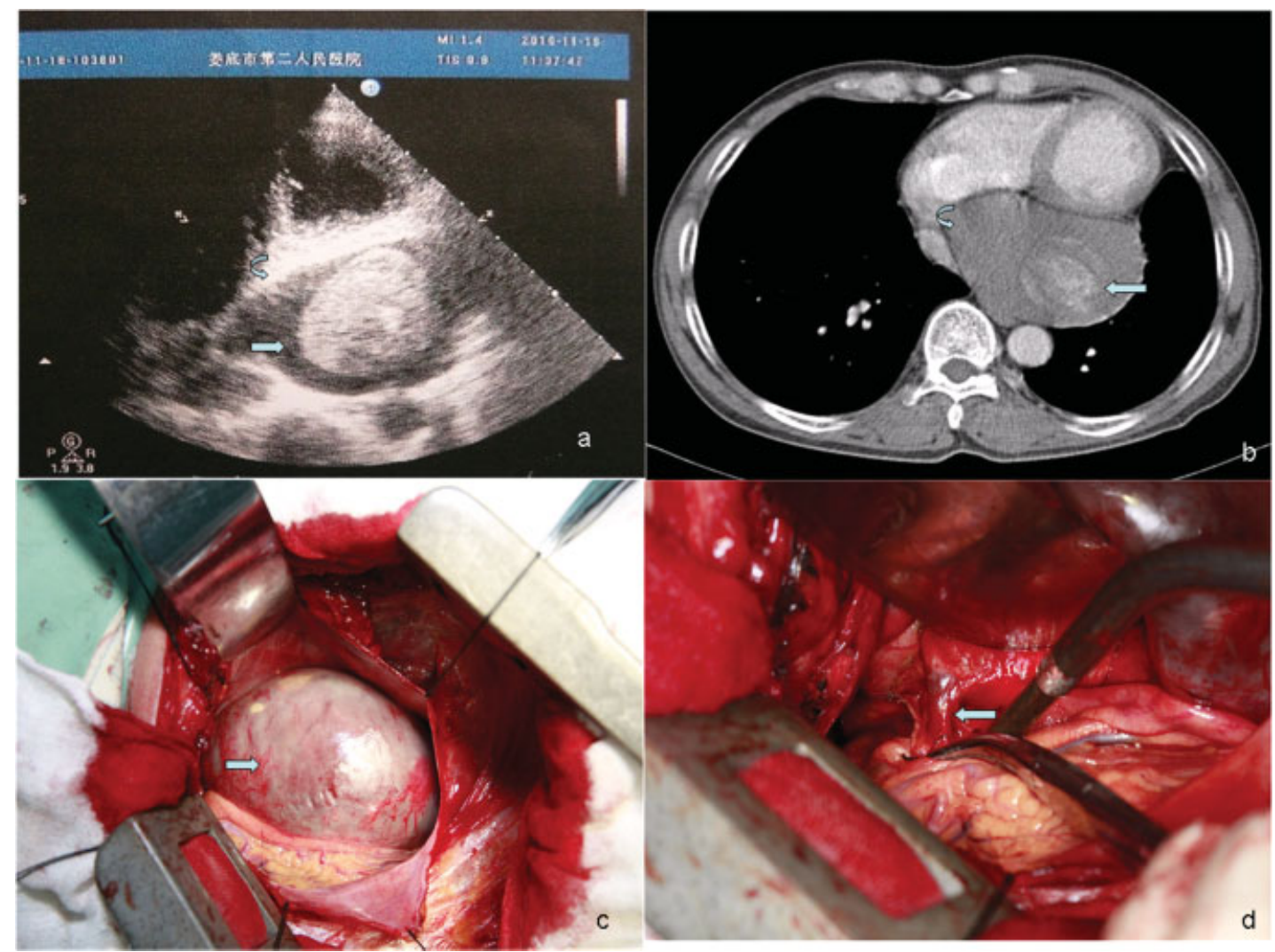

Fig. 1 (a) Color Doppler flow imaging found no blood flow inside the mass. (b) Computed tomography imaging of the mass, confirming no blood flow inside the mass. (c) During the surgery, inside the pericardium, a huge unicamerate cardiac hemangioma in a persistent left superior vena cava was revealed. (d) A communication vein of $3 \mathrm{~mm}$ diameter between the coronary sinus and hemangioma was shown.

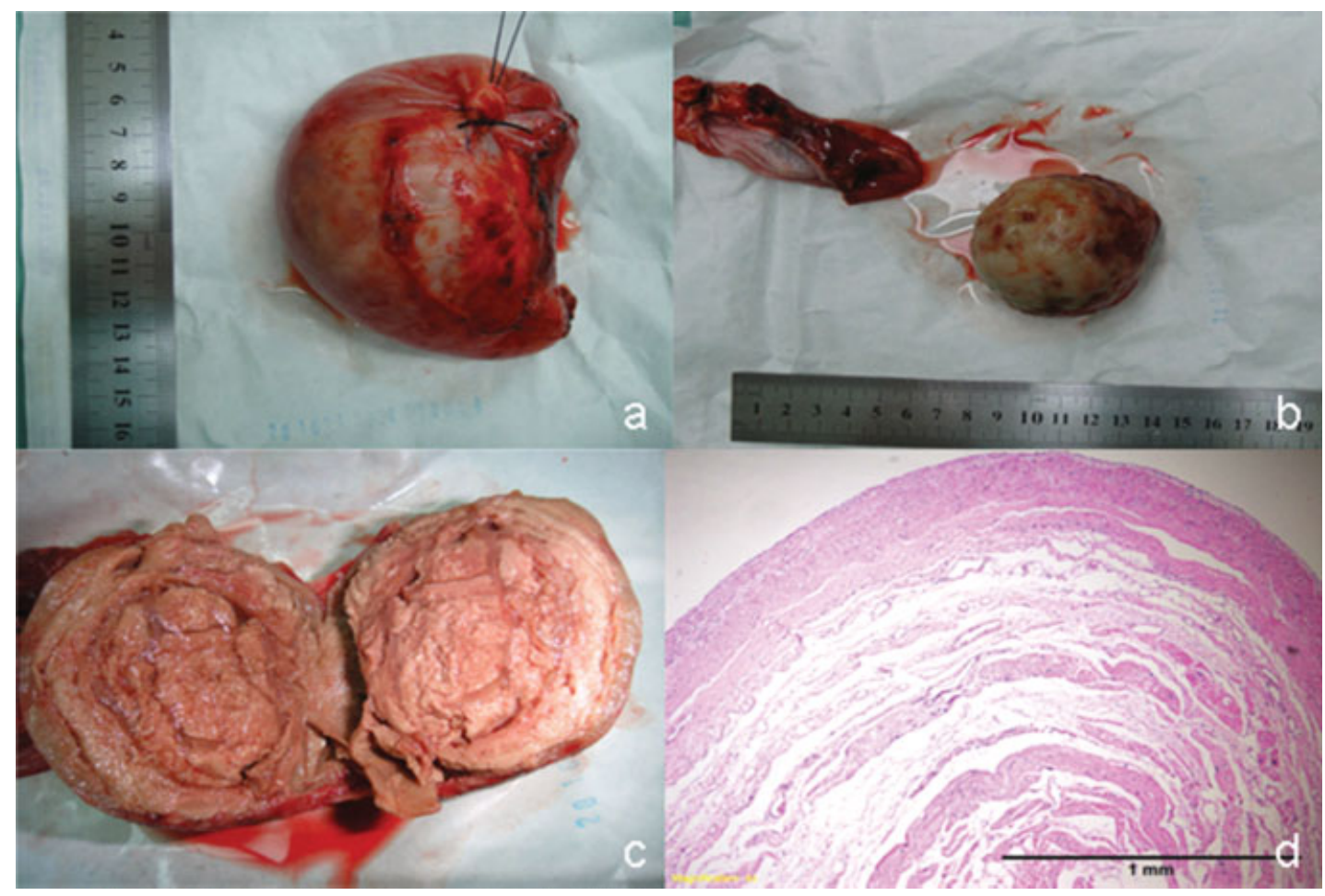

Fig. 2 The tumor measured $110 \times 65 \times 45 \mathrm{~mm}$ with some white spots on the wall (a). Pathology revealed a large venous hemangioma (b) with a huge not-organized onion-like-shaped thrombus core (c). The wall of the mass was composed of flattened endothelial cells, smooth muscle, elastic fibers, and calcified plaques (d) which demonstrated that was an intravenous hemangioma. 
the diagnosis. ${ }^{2}$ Recent developments in three-dimensional imaging and transesophageal echocardiography have further enhanced its role in clinical diagnosis. ${ }^{3}$ CT and magnetic resonance imaging (MRI) were also serviced as conventional methods for further evaluation, such as extension of the tumors and the amount of myocardial involvement of cardiac tumors. ${ }^{4}$ With sophisticated cardiac MRI, early detection and management of these tumors become available. Coronary angiography is sometimes useful to identify the disposition and delineating feeding vessels to the hemangioma before operation. However, as shown in our patient, it is not always easy to distinguish hemangioma from a paracardiac mass.

Once cardiac hemangioma is confirmed, complete resection is the treatment of choice. Whether CPB is needed depends on the relationship between the hemangioma and the heart. Final decision always could be made after a careful exploration during operation. Sacrifice of minor coronary vessel is inevitable but acceptable for resection. Long-term survival improved significantly for most patients operated on with a complete or even an incomplete resection which can release compression and result in resolution of symptoms. Although recurrence rate is low, periodic echocardiography examinations are suggested to monitor for tumor relapse. ${ }^{5}$

The development of thrombosis core is not clear. Based on the findings in operation and histological analysis, we thought the reasons might be as follows: for the hemangioma connected to the coronary sinus by a small communicate vein, the persistent left superior vena outflow was obstructed and the velocity of bloodstream was very low, which was also in accordance with the color Doppler flow imaging; the initial thrombus was formed and detained in the lumen of the persistent left superior vena, then the initial thrombus was rolled by the bloodstream like a "snowball" and developed to a final onion-like-shaped thrombus. Furthermore, the developing rolling thrombus expanded the persistent left superior vena lumen and resulted in the formation of the cardiac hemangioma.

\section{References}

1 Kipfer B, Englberger L, Stauffer E, Carrel T. Rare presentation of cardiac hemangiomas. Ann Thorac Surg 2000;70(3):977-979

2 Sütsch G, Jenni R, von Segesser L, Schneider J. [Heart tumors: incidence, distribution, diagnosis. Exemplified by 20,305 echocardiographies]. Schweiz Med Wochenschr 1991;121(17): 621-629

3 Baldauf J, Müller JU, Fleck S, Hinz P, Chiriac A, Schroeder HW. The value of intraoperative three dimensional fluoroscopy in anterior decompressive surgery of the cervical spine. Zentralbl Neurochir 2008;69(1):30-34

4 Manasse E, Nicolini F, Canziani R, Gallotti R. Left ventricular hemangioma. Eur J Cardiothorac Surg 1999;15(6):864-866

5 Chalet Y, Mace L, Franc B, Neveux JY, Lancelin B. Angiosarcoma 7 years after surgical excision of histiocytoid haemangioma in left atrium. Lancet 1993;341(8854):1217 